\title{
Role of the heme oxygenase/carbon monoxide pathway in the pathogenesis and prevention of hepatic encephalopathy
}

\author{
QIU-MING WANG ${ }^{*}$, XUE-YING YIN* ${ }^{*}$ ZHI-JUN DUAN, SHI-BIN GUO and XIAO-YU SUN \\ Department of Gastroenterology, The First Affiliated Hospital of Dalian Medical University, \\ Dalian, Liaoning 116011, P.R. China
}

Received January 25, 2013; Accepted April 29, 2013

DOI: $10.3892 / \mathrm{mmr} .2013 .1472$

\begin{abstract}
Hepatic encephalopathy (HE) is a severe complication of liver cirrhosis and its pathogenesis has yet to be fully elucidated. Previous studies have demonstrated that heme oxygenase-1 (HO-1) is important in the induction of liver cirrhosis. The present study aimed to investigate the role of HO-1 in the pathogenesis of HE. Rats were divided into 5 treatment groups; sham, bile duct ligation (BDL), HE, zinc protoporphyrin (ZnPP) and cobalt protoporphyrin (CoPP). The levels of HO-1 were examined by western blotting and quantitative real-time PCR (qRT-PCR). Serum levels of carboxyhemoglobin $(\mathrm{COHb})$, ammonia levels in the plasma and brain, brain water content and portal vein pressure (PVP) were also quantified. Aquaporin-4 expression levels were measured by immunohistochemistry and qRT-PCR. The results demonstrated that the levels of HO-1 in the brain and the serum levels of $\mathrm{COHb}$ were significantly increased in the HE group compared with the BDL group. Brain water content, PVP and ammonia levels in the plasma and brain were increased in the HE and CoPP groups; however, these were reduced following the treatment with $\mathrm{ZnPP}$. The levels of AQP-4 expression and oxidative stress in the brain were reduced following treatment with $\mathrm{ZnPP}$ and increased following treatment with CoPP. In conclusion, following the inhibition of HO-1 expression, treatment with $\mathrm{ZnPP}$ improved $\mathrm{HE}$ due to reducing the expression levels of AQP-4 and oxidative stress. Therefore, $\mathrm{ZnPP}$ treatment may represent a novel therapeutic approach for HE.
\end{abstract}

Correspondence to: Professor Zhi-Jun Duan, Department of Gastroenterology, The First Affiliated Hospital of Dalian Medical University, No. 222 Zhongshan Road, Dalian, Liaoning 116011, P.R. China

E-mail: cathydoctor@sina.com

${ }^{*}$ Contributed equally

Key words: heme oxygenase-1, carboxyhemoglobin, aquaporin-4, oxidative stress, hepatic encephalopathy

\section{Introduction}

Hepatic encephalopathy (HE) is a clinical neuropsychiatric syndrome resulting from a metabolic disturbance caused by severe liver disease and/or portal-systemic shunts. HE is associated with significant morbidity and mortality (1). Several theories have been proposed to explain the pathogenesis of HE. One such theory is the ammonia hypothesis, which states that the level of ammonia plays a central role in the pathogenesis of HE. Infection, inflammation, oxidative stress and steroid hormones are also considered to be important (2). As the precise underlying mechanism of the pathogenesis of HE remains unclear and specific treatments for $\mathrm{HE}$ are limited, the reduction of ammonia levels is regarded as the primary approach to prevent HE. Gaining a better understanding of the pathogenesis of $\mathrm{HE}$ is important for the development of effective treatment strategies in the future.

Heme oxygenase-1 (HO-1) catalyzes the oxidative degradation of heme to carbon monoxide $(\mathrm{CO})$, free iron and biliverdin (3). In normal brain tissue, basal HO-1 expression levels and activity are maintained at low levels and are confined to the neuroglia and occasionally, the scattered neurons (4). However, the level of HO-1 activity may be significantly enhanced in astrocytes, microglia and certain neurons by extravascular hemoglobin, hemin and oxidants $(5,6)$. The upregulation of HO-1 attenuates post-ischemic brain damage via simultaneous inhibition of superoxide production and preservation of nitric oxide (NO) bioavailability (7). It has been reported that HO-1 may be a therapeutic target in neurodegenerative disease and brain infection (8).

Our previous studies have indicated that HO-1 expression is upregulated in the liver and the $\mathrm{HO} / \mathrm{CO}$ pathway may regulate cirrhosis induced in rats by bile duct ligation (BDL), as well as its complications, including hepatorenal and hepatopulmonary syndrome $(9,10)$. In addition, the levels of carboxyhemoglobin $(\mathrm{COHb})$ in the arterial blood of hepatitis B virus-related cirrhosis patients with HE were significantly increased compared with healthy individuals (11). The mechanism by which HO-1 is expressed in the brain and affects the development and progression of HE remains unclear.

The present study aimed to evaluate whether the regulation of HO-1 affects the development and progression of HE, and the mechanism by which its products affect cerebral metabolism. 


\section{Materials and methods}

Animal care. The experimental protocols used in the present study were approved by the Animal Care and Use Committee of Dalian Medical University (Liaoning, China) in accordance with the guidelines established by the China Council on Animal Care.

Generation of HE models and the treatment of rats. The 46 healthy male Sprague Dawley rats, weighing 200-220 g, were obtained from the Laboratory Animal Center of Dalian Medical University (Dalian, China) and randomly divided into 5 treatment groups; sham $(\mathrm{n}=6), \mathrm{BDL}(\mathrm{n}=10), \mathrm{HE}(\mathrm{n}=12)$, zinc protoporphyrin ( $\mathrm{nPP} ; \mathrm{n}=8)$ and cobalt protoporphyrin (CoPP; $n=10)$. The rats were housed in a specific pathogen-free center at room temperature $\left(24-26^{\circ} \mathrm{C}\right)$ and a relative humidity of $60-65 \%$. Water was provided ad libitum.

The rats were fed and housed for 3 days prior to any experimental protocols being conducted. All surgical procedures were approved by the Animal Care and Use Committee of Dalian Medical University. Laparotomy was performed under anesthesia with ether. The common bile duct was localized, doubly ligated and cut between these two ligatures. In the sham group, a midline incision was performed in the animals; however, this was without BDL. Four groups underwent BDL and a sham surgery group was used as a control. Two weeks following surgery, the sham and BDL rats were pair-fed and administered with an i.p. saline injection. The HE, ZnPP and CoPP groups were fed on an ammonium-containing diet (ammonium acetate, $20 \% \mathrm{w} / \mathrm{w}$ ), as described previously (12), and were administered with an i.p. saline, ZnPP or CoPP injection $(5 \mathrm{mg} / \mathrm{kg}$ body weight), three times per week, respectively. The animals were treated with $\mathrm{ZnPP}$ and $\mathrm{CoPP}$ in order to downregulate and upregulate the expression of HO-1, respectively. Following the establishment of the HE models, the number of rats in each group was reduced to 6 due to deaths encountered within specific groups.

ZnPP and CoPP (Sigma, St. Louis, MO, USA) were dissolved in $0.2 \mathrm{~mol} / 1 \mathrm{NaOH}$, adjusted to $\mathrm{pH} 7.4$ and diluted in $0.85 \% \mathrm{NaCl}$ to $1 \mathrm{mg} / \mathrm{ml}$, as described previously (13). Histostain $^{\mathrm{TM}}$-Plus (SP9001; Zhongshan Goldenbridge Biological Technology, Beijing, China), superoxide dismutase (SOD) and malonaldehyde (MDA) kits (Nanjing KeyGen Biotech. Co., Ltd., Nanjing, China) were used in the study.

Sample collection and examination. Two weeks following treatment, a catheter connected to a Pressure Transducer (BL-420F biological experimental system; Chengdu Technology and Market Co., Ltd., Chengdu, China) was placed in the portal vein to measure portal vein pressure (PVP). Subsequently, $1 \mathrm{ml}$ of arterial blood was withdrawn to measure serum $\mathrm{COHb}$ levels using a Rapid Lab 1245 Blood Gas Analyzer (Siemens, New York, NY, USA). Levels of alanine aminotransferase (ALT), aspartate aminotransferase (AST), total bilirubin (TBIL) and serum iron were detected using a Hitachi 7600-110 Automatic Biochemical Analyzer (Hitachi Co., Tokyo, Japan).

Measurement of plasma and cerebral ammonia levels. The levels of ammonia were measured in plasma and the cerebral cortex. Blood samples were examined using the Ammonia
Checker II (KEM, Kyoto, Japan). The cerebral ammonia levels were measured by fluorimetry (Fluoroskan Ascent Labsystems, Helsinki, Finland), according to the methods described previously (12). Assays were performed in Costar 96-well UV plates (Corning Costar Corporation, Cambridge, MA, USA).

Measurement of brain water content. The levels of brain water were quantitated by the wet-weight/dry-weight method. Half of the brain was weighed prior to and following $48 \mathrm{~h}$ incubation in a $120^{\circ} \mathrm{C}$ oven. The brain water content percentage was calculated using the following formula: Brain water content $(\%)=($ wet weight - dry weight $) /$ wet weight $\times 100 \%$, as described previously (14).

Measurement of locomotor activity. Locomotor activity was assessed using an infrared beam computerized auto-track system (Columbus Instruments, Columbus, OH, USA) (15). Prior to being sacrificed, rats from each of the 5 groups were individually placed in plexiglass cages $(29 \times 22 \times 22 \mathrm{~cm})$ for $6 \mathrm{~h}$ before activity was recorded. Cumulative distance traveled during the day (inactive period) and night (active period) was recorded for $24 \mathrm{~h}$ and expressed as the night/day ratio.

Measurement of oxidative stress. Levels of brain SOD and MDA were determined using the UV-2100 Spectrophotometer (Chemito Instruments Pvt. Ltd., Mumbai, India), according to the manufacturer's instructions.

Histology and immunohistochemistry. Sections of the liver and brain lobe were excised, fixed in $10 \%$ neutral formalin solution and embedded in paraffin. Hematoxylin and eosin (H\&E) staining was performed according to standard procedure. Lesion severity was graded as described previously (16). Tissue sections (4- $\mu \mathrm{m}$ thick) were briefly treated with $\mathrm{HCl}(5 \%)$ to liberate ferric ions. Samples were then treated with $5 \%$ potassium ferrocyanide to produce insoluble ferric ferrocyanide. Slides were counterstained with neutral red. For immunohistochemical examination, deparaffinized sections were incubated with HO-1 antibodies (Abcam, Cambridge, MA, USA; 1:1,000 dilution), aquaporin-4 (AQP-4) antibodies (Santa Cruz Biotechnology, Inc., Santa Cruz, CA, USA; 1:400 dilution) and biotinylated secondary antibodies (Santa Cruz Biotechnology, Inc.), followed by avidin-biotin-peroxidase complex. Yellow material in the cytoplasm was considered to represent a positive cell. Cell staining was assigned to 4 scores, as described previously (17). The final score was defined as follows: staining intensity x percentage of positive cells. The mean score of 5 fields was used to compare the 5 groups.

Western blot analysis. The resected liver tissues were extracted with lysis buffer ( $1 \%$ Triton X-100, $50 \mathrm{mmol} / \mathrm{l}$ Tris- $\mathrm{HCl} \mathrm{pH} \mathrm{7.6,} 150 \mathrm{mmol} / \mathrm{l} \mathrm{NaCl}$ and $1 \%$ protease inhibitor cocktail). Western blotting was performed in accordance with a previously described protocol (18). Western blot analysis was performed with liver homogenates (30 $\mu \mathrm{g}$ protein) using anti-HO-1 antibody (Abcam; 1:2,000 dilution), anti- $\beta$-actin antibody (Zhongshan Goldenbridge Biological Technology; 1:500 dilution) and secondary anti-rabbit and anti-mouse IgG (Santa Cruz Biotechnology, Inc.; 1:500 dilution). The intensity 
of each signal was corrected by the values obtained from the immunodetection of $\beta$-actin and the relative protein intensity was expressed as the fold-change of the content in the control group.

Quantitative real-time PCR ( $q R T-P C R)$. qRT-PCR was used to analyze the expression levels of HO-1 and AQP-4. Total RNA was extracted from each chamber using RNAiso Plus ((Takara Bio, Inc., Dalian, China). The total RNA was then reverse transcribed into cDNA using the PrimeScript ${ }^{\circledR}$ RT reagent kit (Perfect Real Time; Takara Bio, Inc.). Real-time RT-PCR was performed with a Mx3000P QPCR System (Agilent Technologies, Palo Alto, CA, USA) using SYBR ${ }^{\circledR}$ Premix Ex Taq II (Perfect Real Time). $\beta$-Actin, a common housekeeping gene in cells, was used as the internal control gene to normalize the quantities of target gene expression. Thermocycling conditions were as follows: $95^{\circ} \mathrm{C}$ for $30 \mathrm{sec}$, 40 cycles of denaturation $\left(95^{\circ} \mathrm{C}, 5 \mathrm{sec}\right)$, annealing $\left(60^{\circ} \mathrm{C}\right.$, $30 \mathrm{sec})$ and extension $\left(72^{\circ} \mathrm{C}, 30 \mathrm{sec}\right)$. The primers used in the RT-PCR are listed in Table I.

Statistical analysis. All data are presented as the mean \pm SD. Statistical analysis was performed using SPSS software version 16.0 (IBM, Chicago, IL, USA). Groups were compared using one-way ANOVA with the Dunnett's multiple comparison test (where applicable). $\mathrm{P}<0.05$ was considered to indicate a statistically significant result.

\section{Results}

Diet-induced HE model in BDL rats. Common BDL, ascites and jaundice were observed in BDL rats at 4 weeks post-surgery. The serum levels of AST, ALT and TBIL in the BDL group were significantly higher compared with the sham group $(\mathrm{P}<0.01)$. In addition, the serum levels of AST, ALT and TBIL were significantly increased in the HE group compared with the BDL group $(\mathrm{P}<0.01$; Fig. $1 \mathrm{~B})$.

PVP was significantly higher in the BDL group compared with the sham group $(\mathrm{P}<0.01)$. Compared with the BDL group, the PVP was significantly elevated in HE rats. In addition, PVP decreased following the treatment with $\mathrm{ZnPP}$ and increased following the treatment with CoPP compared with HE rats $(\mathrm{P}<0.01$; Fig. 1Be). Plasma ammonia levels were higher in the BDL group compared with the sham controls. Higher plasma ammonia levels were observed in the HE group compared with the BDL group ( $\mathrm{P}<0.01$; Fig. $1 \mathrm{Cf})$.

Hepatic fibrosis was evaluated by H\&E staining. Compared with the sham group, the BDL and HE groups exhibited increased levels of inflammation, necrosis and destruction of the lobular architecture (Fig. 1A). Brain H\&E staining demonstrated normal cellularity and architecture in the sham group. Compared with the BDL group, HE rats demonstrated more severe cerebral intracellular edema in the brain (Fig. 1B).

Inhibition of HO-1 expression improves $\mathrm{HE}$. The HO-1 mRNA and protein expression levels in the brain increased significantly following BDL treatment compared with the sham controls, and were significantly elevated in the HE group compared with the BDL group $(\mathrm{P}<0.01)$. In addition,
Table I. Primers used for real-time PCR analysis

\begin{tabular}{|c|c|c|}
\hline Gene & $\begin{array}{c}\text { Forward/ } \\
\text { reverse }\end{array}$ & Sequence $5^{\prime}-3^{\prime}$ \\
\hline \multirow[t]{2}{*}{$\mathrm{HO}-1$} & Forward & AGGTGCACATCCGTGCAGAG \\
\hline & Reverse & CTTCCAGGGCCGTATAGATATGGTA \\
\hline \multirow[t]{2}{*}{ AQP-4 } & Forward & TTGGACCAATCATAGGCGC \\
\hline & Reverse & GGTCAATGTCGATCACATGC \\
\hline \multirow[t]{2}{*}{$\beta$-actin } & Forward & GGAGATTACTGCCCTGGCTCCTA \\
\hline & Reverse & GACTCATCGTACTCCTGCTTGCTG \\
\hline
\end{tabular}

HO-1, heme oxygenase-1; AQP-4, aquaporin-4.

the mRNA and protein expression levels of HO-1 were significantly decreased in the ZnPP treatment group compared with the HE group; however, these were enhanced in the CoPP treatment group (Fig. 2B). The $\mathrm{COHb}$ levels in the arterial blood were in accordance with HO-1 expression (Fig. 2Ca). Cerebral HO-1 immunostaining demonstrated that HO-1 was mainly expressed in the cerebral cortex (Fig. 2A).

Decreased levels of AST, ALT and TBIL were observed in the $\mathrm{ZnPP}$ group compared with the HE group; however, these were elevated in the CoPP group (Fig. 1B). The levels of ammonia were lower in the ZnPP treatment group compared with the HE group; however, were higher in the CoPP treatment group $(\mathrm{P}<0.01$; Fig. $1 \mathrm{Cf})$. The levels of ammonia in the brain were significantly higher in the HE group compared with the BDL treatment group; however, these were significantly elevated and reduced in the CoPP and ZnPP treatment groups, respectively, compared with the HE group $(\mathrm{P}<0.01$; Fig. 2Cc).

Liver and brain H\&E staining demonstrated less severe fibrous hyperplasia and cerebral intracellular edema in the brain following treatment with ZnPP; however, these were more severe in the CoPP group (Fig. 1A). The histopathological scores for liver fibrosis were higher in HE and CoPP groups than for those of the ZnPP treatment group (Fig. 1Ad).

Locomotor activity in the BDL group was reduced compared with the sham controls. In addition, the locomotor activity was significantly decreased in the HE group compared with the BDL and CoPP groups $(\mathrm{P}<0.01)$; however, this was normalized following $\mathrm{ZnPP}$ treatment (Fig. 3Bb).

The inhibition of HO-1 expression led to decreased levels of AQP-4 expression and brain edema. The mRNA levels of $\mathrm{AQP}-4$ in the brain were higher in the BDL group compared with the sham group and elevated in the HE group compared with the BDL group $(\mathrm{P}<0.01)$. Compared with the HE group, $\mathrm{AQP}-4$ expression was decreased in the $\mathrm{ZnPP}$ treatment group; however, this was significantly increased in the CoPP treatment group $(\mathrm{P}<0.01$; Fig. 3Ba).

Cerebral AQP-4 immunostaining demonstrated a markedly more intense immunolabeling of the cerebral cortex in the BDL, HE and CoPP treatment groups compared with the sham controls (Fig. 3Aa-e). Quantitative scoring of cerebral AQP-4 protein expression demonstrated that it was reduced in the ZnPP treatment group compared rats in the HE group (Fig. 3Af). 
A

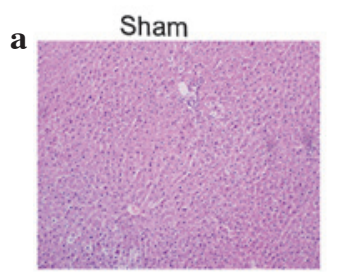

B

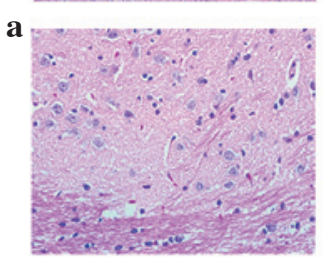

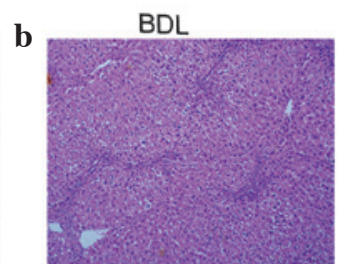

b

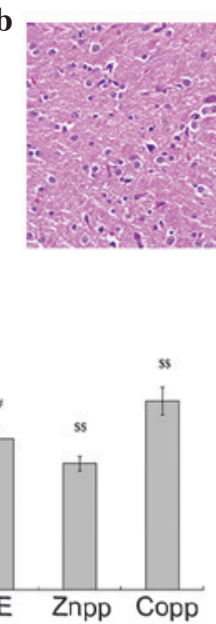

d

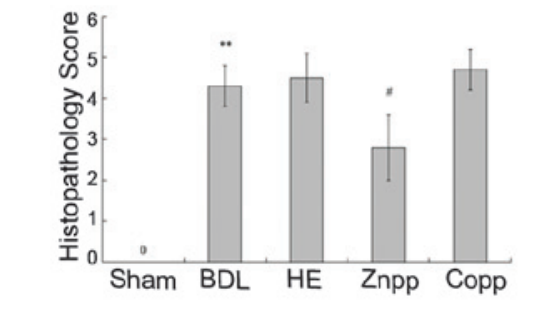

C

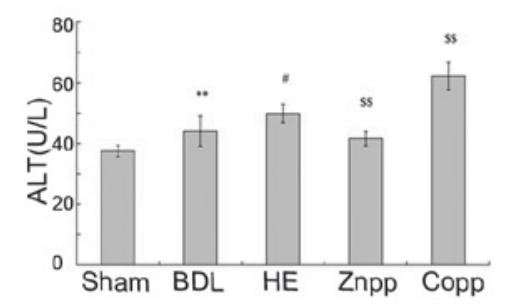

d

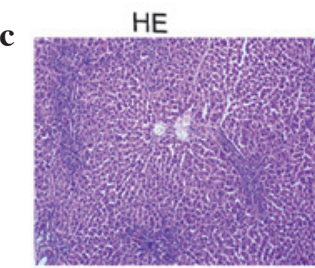

c

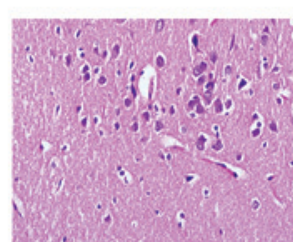

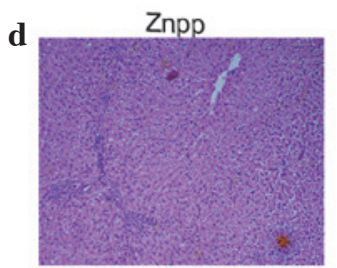

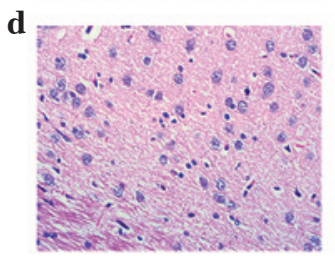

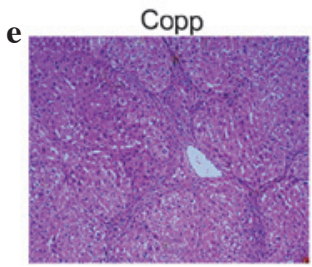

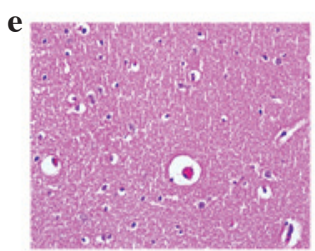

b

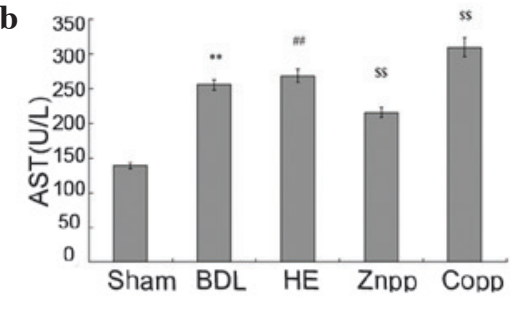

e

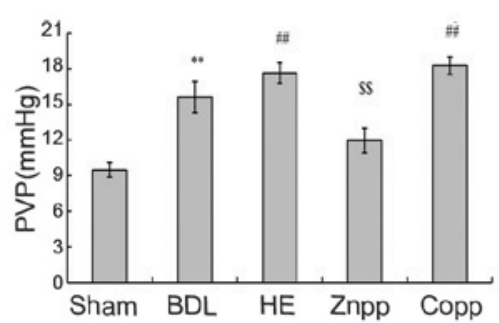

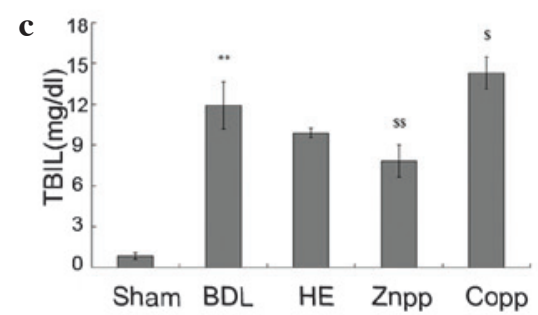

f

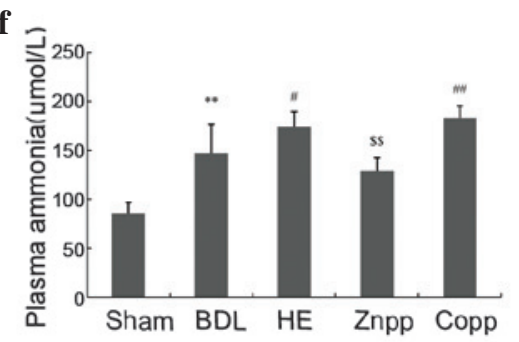

Figure 1. Assessment of HE. (A and B) Liver and brain H\&E staining. (A) Normal lobular architecture observed in the (Aa) sham group; fibrous hyperplasia in the (Ab) BDL, (Ac) HE, (Ad) ZnPP and (Ae) CoPP groups. Less severe fibrous hyperplasia was observed in the ZnPP group compared with the BDL group (original magnification, x100). (Ba) The brain tissue of the sham group demonstrated a normal cellularity and architecture; the cerebral intracellular edema of the brain in the $(\mathrm{Bb}) \mathrm{BDL},(\mathrm{Bc}) \mathrm{HE},(\mathrm{Bd}) \mathrm{ZnPP}$ and $(\mathrm{Be}) \mathrm{CoPP}$ groups. The cerebral intracellular edema was more severe in the HE and CoPP groups compared with the BDL group; however, it was mitigated in the ZnPP group (original magnification, $\mathrm{x} 400$ ). (C) Serum index and PVP; (Ca) ALT, (Cb) AST and (Cc) TBIL levels were significantly increased in the HE and CoPP groups compared with the BDL group; however, they were significantly decreased in the ZnPP group. In addition, the AST, ALT and TBIL levels were lower in the ZnPP group compared with the HE group; (Cd) hepatic fibrosis was assessed using histopathological scoring; (Ce) the PVP was higher in the BDL group compared with the sham group; however, was significantly increased in the HE and CoPP groups compared with the BDL group, and lower in the ZnPP group; (Cf) levels of plasma ammonia were higher in the $\mathrm{HE}$ and CoPP groups compared with the BDL group, and lower in the $\mathrm{ZnPP}$ group. The data are presented as the mean $\pm \mathrm{SD}$. ${ }^{* *} \mathrm{P}<0.01$, ${ }^{*} \mathrm{P}<0.05$ vs. sham; ${ }^{\# \#} \mathrm{P}<0.01,{ }^{\#} \mathrm{P}<0.05$ vs. BDL; ${ }^{\$ \$} \mathrm{P}<0.01,{ }^{\$} \mathrm{P}<0.05$ vs. HE. H\&E, hematoxylin and eosin; HE, hepatic encephalopathy; AST, aspartate aminotransferase; ALT, alanine aminotransferase; TBIL, total bilirubin; BDL, bile duct ligation; CoPP, cobalt protoporphyrin; ZnPP, zinc protoporphyrin; PVP, portal vein pressure.

The water content in the brain significantly increased in the HE group compared with the BDL treatment group. It decreased markedly in the $\mathrm{ZnPP}$ treatment group compared with the HE group $(\mathrm{P}<0.01)$ and was elevated in the CoPP treatment group (Fig. $2 \mathrm{Cb}$ ).

Role of oxidative stress in HE. The levels of oxidative stress in the brain were assessed by measuring the levels of MDA and SOD. The level of MDA was evidently higher in the HE group compared with the BDL group; however, this was lower in the $\mathrm{ZnPP}$ treatment group and higher in the CoPP treatment group compared with the HE group $(\mathrm{P}<0.01$; Fig. $3 \mathrm{Ca})$. The levels of SOD were elevated in the BDL group compared with the sham control $(\mathrm{P}<0.01)$ and were significantly increased in the $\mathrm{ZnPP}$ treatment group compared with the HE group $(\mathrm{P}<0.01)$; however, these were decreased in the CoPP treatment group (Fig. 3Cb).

\section{Discussion}

HE is an extremely common neuropsychiatric disorder observed in patients with advanced liver disease. Current treatment strategies of HE mainly aim to reduce the production and intestinal absorption of ammonia (2). In the present study, we demonstrated that treatment with ZnPP improves $\mathrm{HE}$ and alleviates liver fibrosis induced by a hyperammonemic diet in BDL rats. At 4 weeks, brain edema, hyperammonemia and reduced locomotor activity were observed in the HE group. Compared with the HE group, these indicators were ameliorated in the $\mathrm{ZnPP}$ group; however, were more severe in 
A

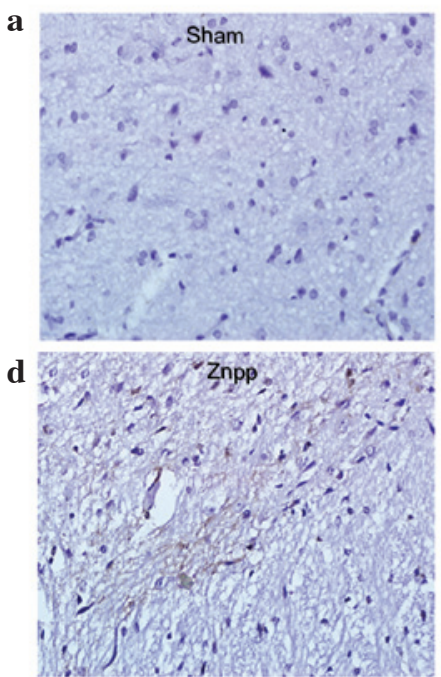

B

a

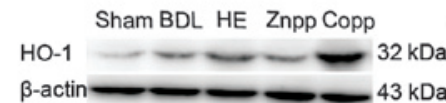

C

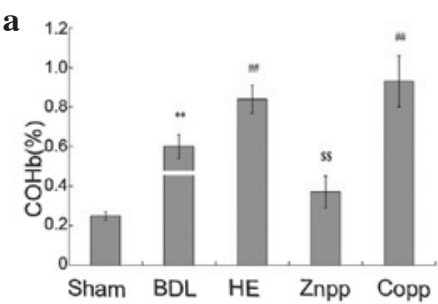

b

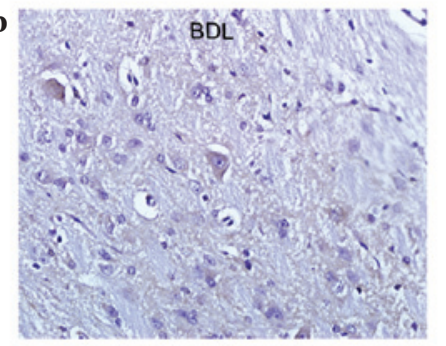

e
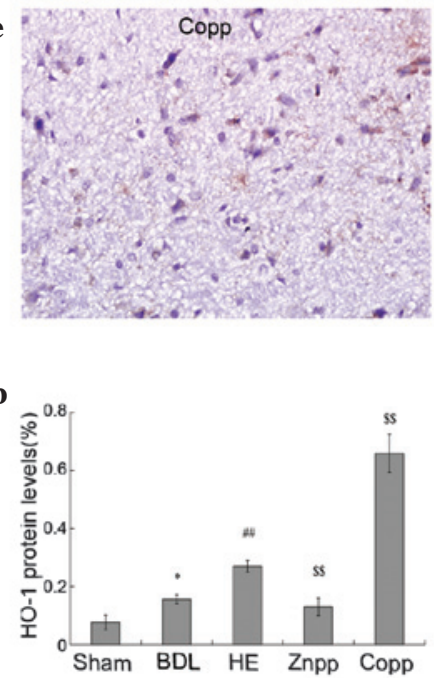

b

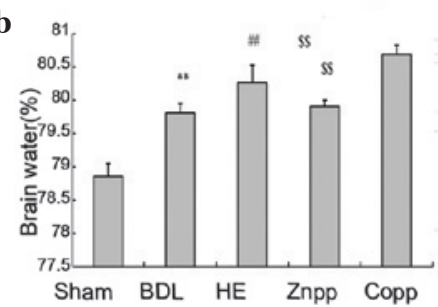

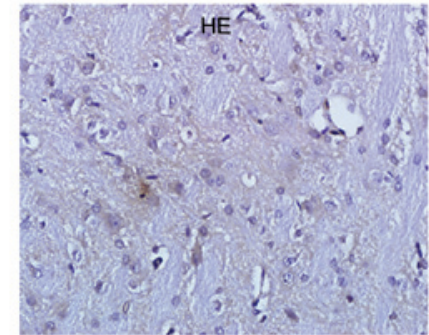

f
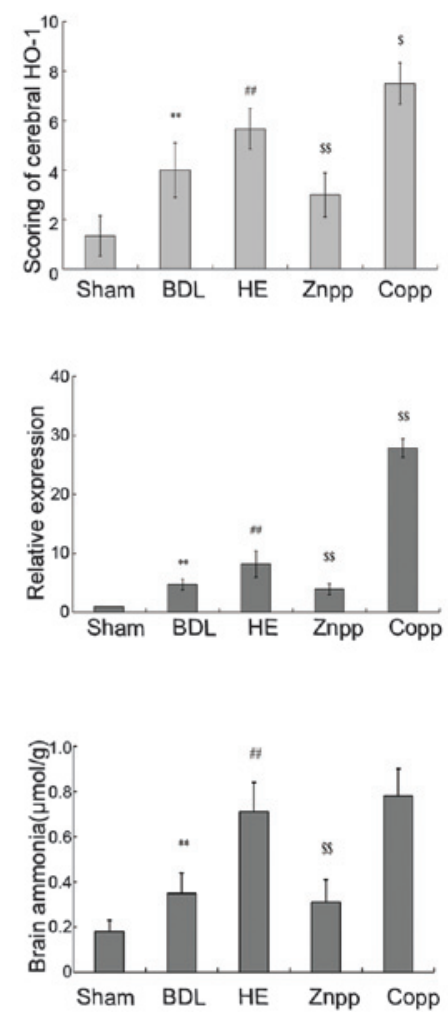

Figure 2. Levels of HO-1, ammonia and brain water content. (A) Immunohistochemical staining with HO-1; (Aa) minimal levels of HO-1 expression were detected in the cerebral cortex in the sham group; HO-1 expression was observed in the (Ab) BDL, (Ac) HE, (Ad) ZnPP and (Ae) CoPP groups. HO-1 was seldomly expressed in the ZnPP group; (Af) quantitative scoring of the immunohistochemical staining of cerebral HO-1 protein expression (original magnification, $\mathrm{x} 400)$. (B) Protein and mRNA expression levels of $\mathrm{HO}-1$; ( $\mathrm{Ba}$ and $\mathrm{Bb}$ ) HO-1 protein expression was higher in the HE and CoPP groups and decreased in the ZnPP group compared with the BDL group; (Bc) HO-1 mRNA levels were quantified using real-time PCR in the brain tissue. (C) Levels of $\mathrm{COHb}$, ammonia and water content. Serum $\mathrm{COHb}$ levels in the arterial blood were quantified. (Ca) Higher levels of $\mathrm{COHb}$ were observed in the $\mathrm{HE}$ and $\mathrm{CoPP}$ groups compared with the BDL group, and were lower in the ZnPP group; $(\mathrm{Cb})$ brain water content and (Cc) ammonia levels in the $\mathrm{HE}$ and CoPP groups were enhanced compared with the BDL group, and decreased in the ZnPP group. The data are presented as the mean $\pm \mathrm{SD}$. ${ }^{* *} \mathrm{P}<0.01$, ${ }^{*} \mathrm{P}<0.05 \mathrm{vs}$. sham; ${ }^{\# \#} \mathrm{P}<0.01$ vs. BDL; ${ }^{\$} \mathrm{P}<0.01,{ }^{\$} \mathrm{P}<0.05$ vs. HE. HO-1, heme oxygenase-1; BDL, bile duct ligation; HE, hepatic encephalopathy; CoPP, cobalt protoporphyrin; ZnPP, zinc protoporphyrin; $\mathrm{COHb}$, carboxyhemoglobin.

the CoPP group. These results demonstrated that HO-1 may be important in the induction of $\mathrm{HE}$ and its inhibition may provide an alternative treatment strategy for HE.

The HO/CO pathway is important in the modulation of microcirculation in normal and cirrhotic conditions (19). However, the mechanism by which it affects the pathogenesis of HE remains unknown. In normal conditions in the central nervous system, the level of HO-1 expression is low (20). Several studies have demonstrated that HO-1 mRNA expression levels are elevated in the frontal cortex of HE rats, which exerts a number of important effects on the coma stage of encephalopathy induced by liver injury $(14,21,22)$. In vitro, HO-1 has been shown to stimulate cytoprotection against oxidative stress (23); however, this protective action has yet to be uniformly observed in vivo. Wang and Doré (24) reported that HO-1 is capable of exacerbating early brain injury following intracerebral hemorrhage. In addition, non-specific $\mathrm{HO}$ inhibitors were able to attenuate brain injury (25). At a specific threshold, HO-1 expression may be beneficial and aid in the protection of hepatocytes (26), whereas HO-1 overexpression may stimulate or aggravate liver cirrhosis (27). The overexpression of HO-1 in the brain cortex of rats acutely intoxicated with ammonia may contribute to cerebral hyperemia in hyperammonemic states (28). The inhibition of bilirubin formation by targeting HO-1 through small interfering RNA (siRNA) may prevent and treat bilirubin encephalopathy at an early clinical stage (29). In the present study, the inhibition of HO-1 was capable of attenuating the levels of serum ammonia and brain edema. Thus, the inhibition of HO-1 may provide an alternative strategy to prevent and treat HE. 
A
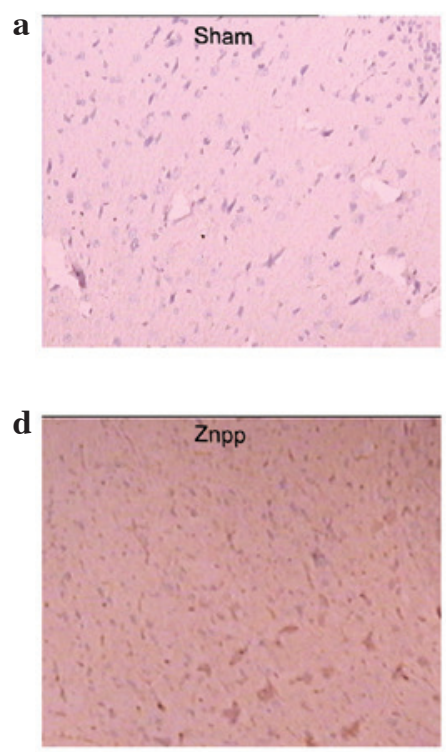

b
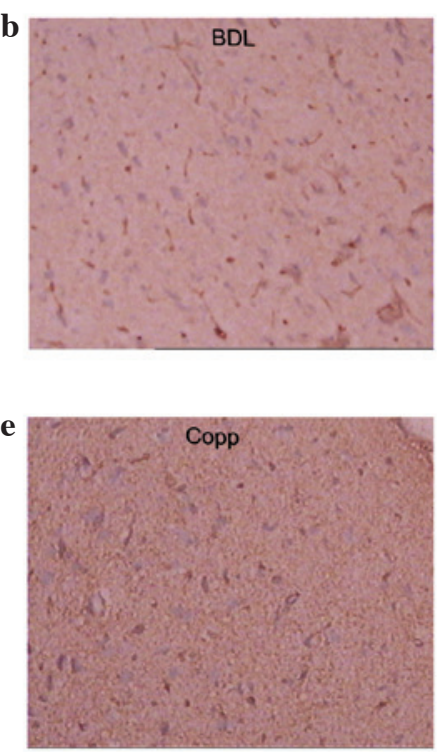

c

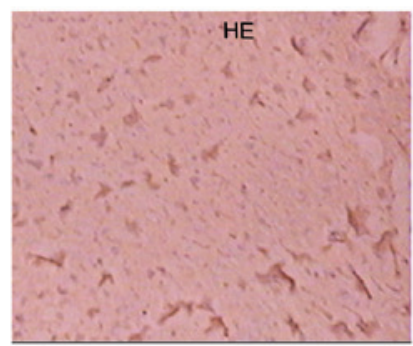

f

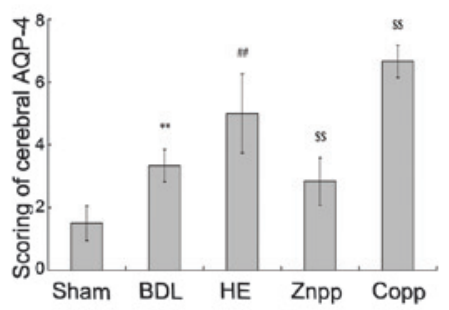

B
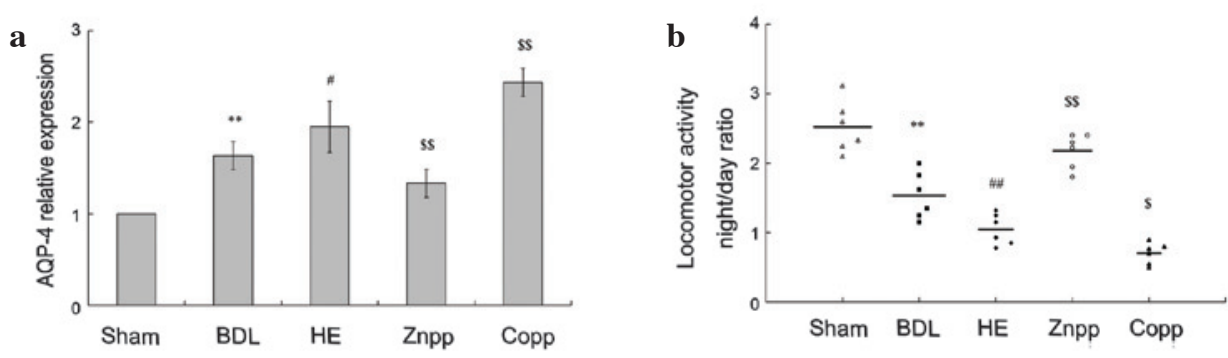

C

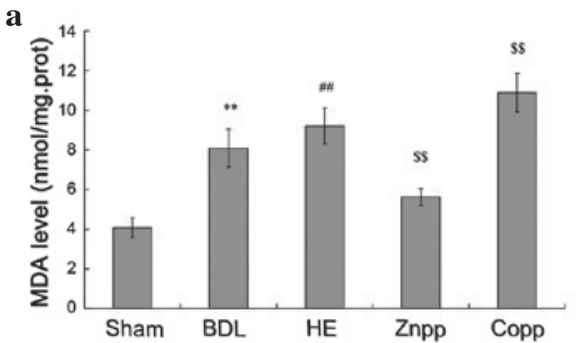

b

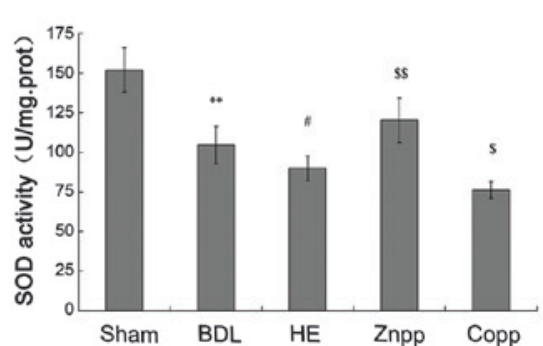

Figure 3. Levels of AQP-4 expression and oxidative stress in the brain. (A) Immunohistochemical staining with AQP-4. Brain sections were stained with AQP-4 antibody; (Aa) AQP-4 was expressed at low levels in the sham group; (Ab, Ac, Ad and Ae) more intense immunolabeling of the brain cerebral cortex was observed in the HE and CoPP groups compared with the BDL group; however, was decreased in the ZnPP group compared with the HE group; (Af) quantitative scoring of immunohistochemical staining of cerebral AQP-4 protein expression (original magnification, $\mathrm{x} 400$ ). (B) AQP-4 mRNA levels and locomotor activity; (Ba) AQP-4 mRNA levels were quantified using real-time PCR in brain tissue. The levels were significantly increased in the HE and CoPP groups compared with the BDL group, and decreased in the ZnPP group; $(\mathrm{Bb})$ locomotor activity was recorded prior to the rats being sacrificed. Data are expressed as the night/day ratio of cumulative distance traveled, recorded over a $12 \mathrm{~h}$ active period (night) and a $12 \mathrm{~h}$ inactive period (day). (C) MDA and SOD levels. Higher MDA and lower SOD levels were detected in the HE and CoPP groups compared with the BDL group. However, the (Ca) MDA levels decreased and (Cb) SOD levels increased significantly in the $\mathrm{ZnPP}$ treatment group. The data are presented as the mean $\pm \mathrm{SD}$. ${ }^{* *} \mathrm{P}<0.01 \mathrm{vs}$. sham; ${ }^{\# \#} \mathrm{P}<0.01,{ }^{\#} \mathrm{P}<0.05 \mathrm{vs}$. $\mathrm{BDL}$; ${ }^{\$ \$} \mathrm{P}<0.01,{ }^{\$} \mathrm{P}<0.05$ vs. HE. AQP-4, aquaporin-4; MDA, malonaldehyde; SOD, superoxide dismutase; HE, hepatic encephalopathy; CoPP, cobalt protoporphyrin; ZnPP, zinc protoporphyrin; BDL, bile duct ligation.

The predominant endogenous source of $\mathrm{CO}$ in the body derives from the degradation of heme catalyzed by HO-1, forming $\mathrm{CO}$, biliverdin and free iron molecules $(30,31)$. $\mathrm{COHb}$ levels may be used to estimate the activity of $\mathrm{HO}$ in experimental animals (32). Our previous studies have demonstrated that the level of $\mathrm{COHb}$ is higher in cirrhotic patients compared with healthy individuals and patients with chronic hepatitis. These findings have additionally been reported in several other studies (33-35). The overexpression of $\mathrm{CO}$ leads to the over-expansion of splanchnic vessels, a decrease in vascular resistance and an increase in blood volume, leading to the hyperdynamic circulation of hepatic cirrhosis with portal hypertension $(36,37)$. In the present study, serum $\mathrm{COHb}$ levels increased in the HE and CoPP treatment groups with portal hypertension and brain injury. However, HE was alleviated in the $\mathrm{ZnPP}$ treatment group, which demonstrated lower levels of serum COHb. This may be due to the inhibition of HO-1 expression decreasing PVP by regulating the $\mathrm{CO} / \mathrm{NO}$ pathway and reducing the levels of toxic substances transported into the brain. The HO/CO pathway may be involved in the pathogen- 
esis of HE in addition to a number of other known mechanisms. Previous studies have demonstrated that the simultaneous inhibition of $\mathrm{NO}$ and $\mathrm{HO}$ completely reverses the reduction in the vasoconstrictor response to potassium chloride in the mesenteric vascular bed (36). We hypothesize that splanchnic vasodilation presenting in portal hypertension is likely to be multifactorial in origin and stimulated in part by an excessive release of $\mathrm{NO}, \mathrm{CO}$ and several other vasoactive mediators.

Free iron is a byproduct of the degradation of heme catalyzed by HO-1. Free iron molecules are capable of participating in the Fenton and Haber-Weiss reactions, and excessive redox-active iron may lead to oxidative stress and the subsequent damage of membranes, proteins and DNA (38). Notably, the overexpression of HO-1 did not lead to an overload of iron in the brain cortex and other anatomical sites, indicated in our study by the Perl's Prussian blue stain (data not shown). This was due to iron primarily accumulating in reticuloendothelial cells, including the spleen, liver and bone. In addition, the overproduction of iron may transfer to other organs apart for the brain, avoiding injury to cerebral cells.

Brain edema is an important component of HE that is associated with acute liver failure. The edema is mainly due to the swelling of astrocytes (cytotoxic edema). Ammonia and AQP-4 are abundantly expressed in astrocytes and may be involved in the development of edema (39). AQP-4 is the predominant water channel expressed in the brain and is distributed widely in brain tissue, with the exception of neurons. It has been demonstrated that AQP-4 is important in maintaining water homeostasis in the brain tissue and participates in super-acute ischemic brain edema $(40,41)$. Early cytotoxic brain edema following brain injury in addition to a secondary insult or focal ischemia results in a vasopressin $\mathrm{V}$ receptor-mediated response, most likely through the upregulation of AQP-4 (42). In the present study, the expression levels of AQP-4 and HO-1 were increased in the HE and BDL groups. The overexpression of HO-1 in the CoPP treatment group led to a high brain water content, which may be correlated with the activation of AQP-4 by ammonia. By contrast, lower levels of ammonia in plasma due to low PVP in the ZnPP treatment group led to the inhibition of AQP-4 expression and a reduction in the severity of brain edema.

Several studies have demonstrated an increased level of oxidative/nitrosative stress in the brain of liver failure patients and its reduction has been shown to be beneficial in animal models (43). Oxidative/nitrosative stress is important in ammonia-induced cell swelling in cultured cells and SOD inhibits the swelling of astrocytes. Wang et al (24) demonstrated that HO-1 knockout mice sustain less severe brain injury and exhibit less neurological dysfunction compared with wild-type mice during the early stages following intracerebral hemorrhage. Previous studies have demonstrated that excess iron is able to participate in the Fenton and Haber-Weiss reactions, leading to oxidative stress and subsequent damage to cell membranes, proteins and DNA (38). In the present study, the upregulation of HO-1 expression increased the levels of oxidative stress, which may be due to free iron produced from the degradation of heme catalyzed by HO-1. Inhibition of HO-1 in the ZnPP treatment group led to lower levels of oxidative stress in the brain and a notable reduction in the levels of neurocyte injury.
In conclusion, we demonstrated that the inhibition of HO-1 expression is capable of attenuating $\mathrm{HE}$ by inhibiting the expression of APQ-4 and reducing the levels of oxidative stress, while decreasing PVP and improving liver fibrosis in BDL rats. The results additionally demonstrated that the $\mathrm{HO} / \mathrm{CO}$ pathway is important in the inhibition of HO-1 expression, which may prevent $\mathrm{HE}$, and elucidate the role of high levels of $\mathrm{COHb}$ in cirrhosis patients with $\mathrm{HE}$ (11). Brain biopsies are rarely performed in patients with HE due to the temporary nature of the disease and surgery often not being necessary; therefore, the animal experiment provides an appropriate supplement to the cerebral pathology of patients with HE. In addition, we demonstrated that HO-1 is important in a number of organs involved in end-stage liver disease $(9,10)$. Thus, additional research with selective HO inhibitors and their doses is required. Effectively regulating the levels of HO-1 during liver cirrhosis may be beneficial. In addition, we hypothesize that a reduction in the levels of $\mathrm{CO}$ resulting from the inhibition of $\mathrm{HO}-1$ may provide a novel therapeutic strategy for decreasing PVP and reducing plasma ammonia levels. The $\mathrm{HO} / \mathrm{CO}$ pathway is involved in the regulation of $\mathrm{HE}$ pathogenesis and may therefore provide a novel therapy for $\mathrm{HE}$.

\section{Acknowledgements}

This study was supported by grants from the National Natural Science Foundation of China (no. 30970886), the Science, Technology Project of Dalian (no. 2010E15SF179) and the Doctoral Initial Funding of Liaoning province (no. 20121110).

\section{References}

1. Ferenci P, Lockwood A, Mullen K, Tarter R, Weissenborn K and Blei AT: Hepatic encephalopathy - definition, nomenclature, diagnosis, and quantification: final report of the working party at the 11th World Congresses of Gastroenterology, Vienna, 1998. Hepatology 35: 716-721, 2002.

2. Atluri DK, Prakash R and Mullen KD: Pathogenesis, diagnosis, and treatment of hepatic encephalopathy. J Clin Exp Hepatol 2: 77-86, 2011.

3. Sass G, Barikbin R and Tiegs G: The multiple functions of heme oxygenase-1 in the liver. Z Gastroenterol 50: 34-40, 2012.

4. Barañano DE and Snyder SH: Neural roles for heme oxygenase: contrasts to nitric oxide synthase. Proc Natl Acad Sci USA 98: 10996-11002, 2001.

5. Turner CP, Bergeron M, Matz P, et al: Heme oxygenase-1 is induced in glia throughout brain by subarachnoid hemoglobin. J Cereb Blood Flow Metab 18: 257-273, 1998.

6. Matz PG, Weinstein PR and Sharp FR: Heme oxygenase-1 and heat shock protein 70 induction in glia and neurons throughout rat brain after experimental intracerebral hemorrhage. Neurosurgery 40: 152-160, 1997.

7. Chao XD, Ma YH, Luo P, et al: Up-regulation of heme oxygenase- 1 attenuates brain damage after cerebral ischemia via simultaneous inhibition of superoxide production and preservation of NO bioavailability. Exp Neurol 239: 163-169, 2013.

8. Cuadrado A and Rojo AI: Heme oxygenase-1 as a therapeutic target in neurodegenerative diseases and brain infections. Curr Pharm Des 14: 429-442, 2008.

9. Guo SB, Duan ZJ, Li Q and Sun XY: Effect of heme oxygenase-1 on renal function in rats with liver cirrhosis. Shi Jie Wei Chang Bing Xue Za Zhi Bian Ji Bu 17: 322-328, 2011 (In Chinese).

10. Guo SB, Duan ZJ, Li Q and Sun XY: Effects of heme oxygenase-1 on pulmonary function and structure in rats with liver cirrhosis. Zhong Hua Yi Xue Za Zhi Bian Ji Bu 124: 918-922, 2011 (In Chinese). 
11. Sun XY, Duan ZJ, Li YL and Chang QS: Detection of carboxyhemoglobin in patients with hepatic encephalopathy due to hepatitis B virus-related cirrhosis. Zhong Hua Yi Xue Za Zhi Bian Ji Bu 125: 3991-3996, 2012 (In Chinese).

12. Jover R, Rodrigo R, Felipo V, et al: Brain edema and inflammatory activation in bile duct ligated rats with diet-induced hyperammonemia: A model of hepatic encephalopathy in cirrhosis. Hepatology 43: 1257-1266, 2006.

13. Amersi F, Buelow R, Kato H, Ke B, Coito AJ, Shen XD, Zhao D, Zaky J, Melinek J, Lassman CR, Kolls JK, Alam J, Ritter T, Volk HD, Farmer DG, Ghobrial RM, Busuttil RW and Kupiec-Weglinski JW: Upregulation of heme oxygenase-1 protects genetically fat Zucker rat livers from ischemia/reperfusion injury. J Clin Invest 104: 1631-1639, 1999.

14. Jiang W, Desjardins P and Butterworth RF: Hypothermia attenuates oxidative/nitrosative stress, encephalopathy and brain edema in acute (ischemic) liver failure. Neurochem Int 55: 124-128, 2009.

15. Butterworth RF, Lalonde R, Power C, Baker GB, Gamrani H and Ahboucha S: Dehydroepiandrosterone sulphate improves cholestasis-associated fatigue in bile duct ligated rats. Neurogastroenterol Motil 21: 1319-1325, 2009.

16. Shackelford C, Long G, Wolf J, Okerberg C and Herbert R: Qualitative and quantitative analysis of nonneoplastic lesions in toxicology studies. Toxicol Pathol 30: 93-96, 2002.

17. Remmele W and Stegner HE: Recommendation for uniform definition of an immunoreactive score (IRS) for immunohistochemical estrogen receptor detection (ER-ICA) in breast cancer tissue. Pathologe 8: 138-140, 1987 (In German).

18. Kakinuma S, Tanaka Y, Chinzei R, et al: Human umbilical cord blood as a source of transplantable hepatic progenitor cells. Stem Cells 21: 217-227, 2003.

19. Van Landeghem L, Laleman W, Vander Elst I, et al: Carbon monoxide produced by intrasinusoidally located haem-oxygenase-1 regulates the vascular tone in cirrhotic rat liver. Liver Int 29: 650-660, 2009

20. Chopra VS, Chalifour LE and Schipper HM: Differential effects of cysteamine on heat shock protein induction and cytoplasmic granulation in astrocytes and glioma cells. Brain Res Mol Brain Res 31: 173-184, 1995

21. Jiang W, Desjardins P and Butterworth RF: Minocycline attenuates oxidative/nitrosative stress and cerebral complications of acute liver failure in rats. Neurochem Int 55: 601-605, 2009.

22. Chastre A, Jiang W, Desjardins P and Butterworth RF: Ammonia and proinflammatory cytokines modify expression of genes coding for astrocytic proteins implicated in brain edema in acute liver failure. Metab Brain Dis 25: 17-21, 2010.

23. Chen K, Gunter K and Maines MD: Neurons overexpressing heme oxygenase-1 resist oxidative stress-mediated cell death. J Neurochem 75: 304-313, 2000.

24. Wang J and Doré S: Heme oxygenase-1 exacerbates early brain injury after intracerebral haemorrhage. Brain 130: 1643-1652, 2007.

25. Koeppen AH, Dickson AC and Smith J: Heme oxygenase in experimental intracerebral hemorrhage: the benefit of tin-mesoporphyrin. J Neuropathol Exp Neurol 63: 587-597, 2004.

26. Malaguarnera L, Madeddu R, Palio E, Arena N and Malaguarnera M: Heme oxygenase-1 levels and oxidative stress-related parameters in non-alcoholic fatty liver disease patients. J Hepatol 42: 585-591, 2005.
27. Froh M, Conzelmann L, Walbrun P, et al: Heme oxygenase-1 overexpression increases liver injury after bile duct ligation in rats. Shi Jie Wei Chang Bing Xue Za Zhi Bian Ji Bu 13: 3478-3486, 2007 (In Chinese).

28. Warskulat U, Görg B, Bidmon HJ, Müller HW, Schliess F and Häussinger D: Ammonia-induced heme oxygenase-1 expression in cultured rat astrocytes and rat brain in vivo. Glia 40: 324-336, 2002.

29. Xia ZW, Li CE, Jin YX, et al: Reduction of bilirubin by targeting human heme oxygenase-1 through siRNA. Exp Biol Med (Maywood) 232: 495-502, 2007.

30. De las Heras D, Fernändez J, Ginès P, et al: Increased carbon monoxide production in patients with cirrhosis with and without spontaneous bacterial peritonitis. Hepatology 38: 452-459, 2003.

31. Tran TT, Martin P, Ly H, Balfe D and Mosenifar Z: Carboxyhemoglobin and its correlation to disease severity in cirrhotics. J Clin Gastroenterol 41: 211-215, 2007.

32. Carter EP, Hartsfield CL, Miyazono M, Jakkula M, Morris KG Jr, and McMurtry IF: Regulation of heme oxygenase-1 by nitric oxide during hepatopulmonary syndrome. Am J Physiol Lung Cell Mol Physiol 283: L346-L353, 2002.

33. Liu J, Duan J, Zhu L, Yang D, Wang L and Shao H: The preliminary study of the relationship between oxygenase-carbon monoxide system and plasma endothelin in hepatic cirrhotic patients with portal hypertension. Zheng Zhou Da Xue 9: 745-748, 2008 (In Chinese).

34. Tarquini R, Masini E, La Villa G, et al: Increased plasma carbon monoxide in patients with viral cirrhosis and hyperdynamic circulation. Am J Gastroenterol 104: 891-897, 2009.

35. Chen YC, Ginès P, Yang J, et al: Increased vascular heme oxygenase-1 expression contributes to arterial vasodilation in experimental cirrhosis in rats. Hepatology 39: 1075-1087, 2004.

36. Fernandez M, Lambrecht RW and Bonkovsky HL: Increased heme oxygenase activity in splanchnic organs from portal hypertensive rats: role in modulating mesenteric vascular reactivity. J Hepatol 34: 812-817, 2001

37. Angermayr B, Mejias M, Gracia-Sancho J, Garcia-Pagan JC, Bosch J and Fernandez M: Heme oxygenase attenuates oxidative stress and inflammation, and increases VEGF expression in portal hypertensive rats. J Hepatol 44: 1033-1039, 2006.

38. Pietrangelo A: Metals, oxidative stress, and hepatic fibrogenesis. Semin Liver Dis 16: 13-30, 1996.

39. Rama Rao KV and Norenberg MD: Aquaporin-4 in hepatic encephalopathy. Metab Brain Dis 22: 265-275, 2007.

40. Papadopoulos MC and Verkman AS: Aquaporin-4 and brain edema. Pediatr Nephrol 22: 778-784, 2007.

41. Badaut J, Ashwal S, Adami A, et al: Brain water mobility decreases after astrocytic aquaporin-4 inhibition using RNA interference. J Cereb Blood Flow Metab 31: 819-831, 2011.

42. Kleindienst A, Dunbar JG, Glisson R and Marmarou A: The role of vasopressin V1A receptors in cytotoxic brain edema formation following brain injury. Acta Neurochir (Wien) 155: 151-164, 2012.

43. Bemeur C, Desjardins P and Butterworth RF: Evidence for oxidative/nitrosative stress in the pathogenesis of hepatic encephalopathy. Metab Brain Dis 25: 3-9, 2010. 